\title{
The effect of hunger on the threshold of behavioral arousal'
}

\author{
ROBERT C. BOLLES, UNIVERSITY OF WASHINGTON \\ MARY SUE YOUNGER, VIRGINIA POLYTECHNIC INSTITUTE
}

The threshold of behavioral arousal in the rat was measured by presenting a series of bursts of noise of increasing intensity, and observing S's behavior. Such thresholds were found to be related to ongoing behavior, history of testing, and conditions of deprivation. A single severe deprivation had no effect upon the threshold but regularly scheduled feeding lowered it.

Campbell \& Sheffield (1953) have suggested that the chief effect of food deprivation is to lower the organism's threshold of responsiveness to stimulation. The evidence bearing on this hypothesis is difficult to evaluate, however, since most of it is based upon general activity measures which present a number of interpretive problems. The purpose of this paper is to describe a method for testing the CampbellSheffield hypothesis more directly, and to present some findings that may help clarify it.

Method

The Ss were 80 nafve Sprague-Dawley rats, predominantly females, about 75 days old. They were tested eight at a time. Each Swas housed in an ordinary hanging cage which had the front wall of hardware cloth replaced by Plexiglas. The cages were placed in a rack and the rack was located in a soundproof isolation room and viewed through a one-way window from a soundproof antechamber.

The test stimuli were $0.5 \mathrm{sec}$. bursts of high frequency white noise produced by a Scientific Prototype generator and attenuator and presented by a University T-202 tweeter. Sound levels were measured at the front of the test cages. An exhaust fan in the isolation room produced a background sound level of $53 \mathrm{~dB}$. All Ss were maintained on a 12-hr. light, 12-hr. dark cycle, the dark being produced by low wattage red lights, and the light by an overhead 32-W fluorescent light.

The observer recorded each S's ongoing behavior, pressed a button to initiate the $0.5 \mathrm{sec}$. test noise, and recorded which Ss responded to it. No distinction was made between startle, disruption of ongoing behavior, and a full display of orienting and exploratory behavior; all were classed as behavioral arousal. The test noise was initially at a low level, typically 25 to $30 \mathrm{~dB}$, and was presented at approximately 1-min. intervals in $5 \mathrm{~dB}$ increments up to a level of 80 to 85 dB. Any $S$ not responding at that point was arbitrarily credited with a threshold $5 \mathrm{~dB}$ higher than the highest test intensity.

In the first experiment a series of test noises was presented every $2 \mathrm{hr}$. over a $48-\mathrm{hr}$. period.
This procedure was then 'repeated with a second group of eight Ss so that the median threshold for a given time of day was based upon 32 individual threshold determinations.

The second experiment was run to assess possible adaptation to the test stimuli. The procedure was the same as before except that only 10 test series were run, and they were spaced every $30 \mathrm{~min}$. This was a faster rate of testing than any we intended to use, so it was expected to indicate an upper limit for the magnitude of adaptation effects to be found elsewhere. Three replications were run.

All of the above Ss had been ad lib food and water. In the third experiment half of the Ss were ad lib and half were totally deprived of food for seven days, resulting in $30 \%$ or more weight loss. Two replications were run. In the fourth experiment half of the Ss were ad $\mathrm{llb}$ but the other half were fed a limited ration regularly once a day. The ration was about $8 \mathrm{gm}$ resulting in a 15\% weight loss. Testing started after Ss had been on the regimen two or three days and continued for 10 days. Three replications were run. All Ss were given two tests a day, $90 \mathrm{~min}$. apart. The daily feeding $\mathrm{Ss}$ were tested $30 \mathrm{~min}$. before the scheduled feeding and $60 \mathrm{~min}$. after, by which time they had nearly always finished eating.

\section{Resulfs}

The median arousal threshold followed a large, approximately sinusoidal cycle; the threshold was roughly $40 \mathrm{~dB}$ lower in the middle of the night than it was in the middle of the day. However, it turns out that the rat is more easily aroused at night, not because of any drive or arousal factor per se, but simply because it is more apt to be awake at that time. When the data were segregated according to the behavior occurring at the time of testing, i.e., according to whether $S$ was asleep or lying still on one hand, or exploring, eating, grooming, on the other, the diurnal cycle of arousability disappeared. This analysis leaves no indication of a $24 \mathrm{hr}$. cycle in the threshold of either sleeping or waking Ss. The threshold of sleeping Ss was typically about $10 \mathrm{~dB}$ higher than those tested while lying still and about $60 \mathrm{~dB}$ higher than Ss that were eating, grooming, or exploring. No consistent differences were found among the thresholds for the different kinds of waking activities.

There were also marked individual differences among Ss in overall threshold, but these too disappeared when the scores were broken down according to the wakesleep state; the superficial individual differences in arousal appeared to be only differences in the overall 
percentage of time different Ss were asleep or awake. Henceforth, all threshold data will be analyzed in this way rather than being presented just as overall means or medians.

The results of the second experiment in which test stimuli were presented every $30 \mathrm{~min}$. indicated a gradual, approximately linear loss of sensitivity over the 10 tests amounting to 10 to $15 \mathrm{~dB}$. Comparable losses were found for sleeping and awake Ss.

The results for the hungry and control Ss are shown in Figs. 1 and 2. Since testing was done shortly after IIghts-off, there was relatively little sleeping, and It was necessary to pool all the sleep data over days of testing, hence the isolated points in Fig. 1.

The thresholds of continuously deprived and control Ss were not significantly different either while asleep or while awake, nor was there a difference in the incidence of wakefulness at the time of testing. On the other hand, a comparison of the regularly fed and control Ss (the fourth experiment) indicated significantly lower thresholds for the hungry Ss before the regularly scheduled meal. Goodman's (1954) modified Kolmogorov-Smirnov test yielded p's of .001 for sleeping and .01 for waking Ss. After eating the corresponding p's were .30 and .05 . These differences would be magnified by an overall comparison,

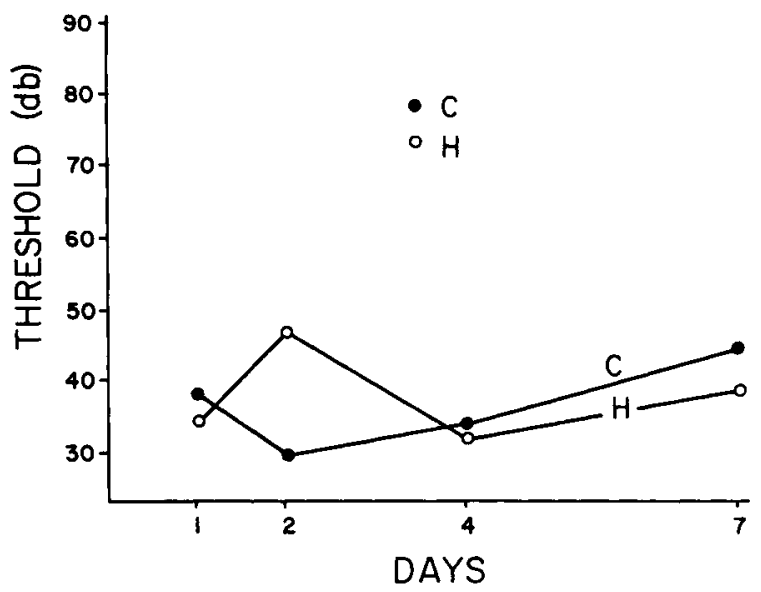

Fig. 1. Median threshold for 8 hungry (H) and 8 control (C) Ss tested twice a day and never fed.

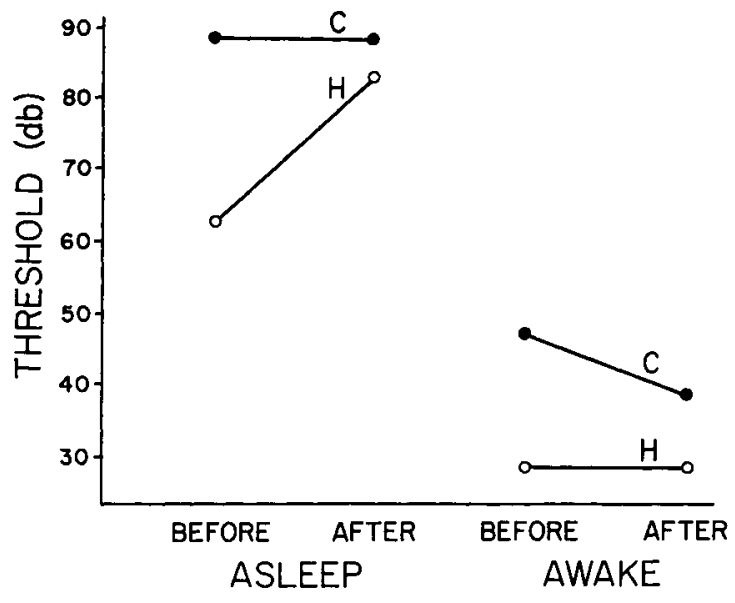

Fig. 2. Median threshold for 12 hungry and 12 control Ss tested before and after regular feeding.

since the hungry Ss were twice as likely as the controls to be awake at the time of testing.

Discussion

These observations illustrate a potentially powerful method for investigating motivational phenomena. The results on the threshold of behavioral arousal are entirely consistent with a growing mass of evidence which suggests that deprivation alone has relatively little effect in motivating an animal. It seems that animals become motivated not so much because we deprive them as because we feed them. It is when $S$ is regularly fed that he becomes responsive, alert, and comes, in short, to act like a motivated animal. Such findings suggest that the hypothetical motivating state called arousal should be conceived to be more like incentive than like drive.

\section{References}

Campbell, B. A., \& Sheffield, F. D. Relation of random activity to feed deprivation. J. comp. physiol. Psychol., 1953, 46, 320322 .

Goodman, L. A. Kolmogorov-Smirnov tests for psychological research. Psychol. Bull., 1954, 51, 160-168.

\section{Note}

1. This research was supported by grant NSG-396 from NASA. 